\title{
D Design and Implementation of Limestone Activity Real-time Detection System Based on Qt /Qwt Hao XU ${ }^{1}$, a , Pingping SHAN ${ }^{1}, \mathrm{Ke} \mathrm{CHEN}^{1}$, Zheng ZHANG ${ }^{1}$
}

${ }^{1}$ School of Software ,Nanyang Institute of Technology, He Nan,Nan Yang, 473004, China aemail: xuhao_hl@163.com

Keywords: limestone;PH value detection;real-time

\begin{abstract}
The limestone Detection System is developed by Qt , assisted by Qwt and Modbus protocol, based on Linux platform. The system can detect activity of limestone and PH value in real-time. This article begins with a brief description of the background and the functional requirements of the system, given the overall design; then introduces Qt and Qwt development library ; finally explaines the key technologies of the modules. The system meets the national standard of limestone detection, has many features, such as stable and reliable, fast response, and has friendly interface, easy to operate and so on.
\end{abstract}

\section{Introduction}

With increasing demand for industrial control, detection of limestone activity and $\mathrm{pH}$ has become increasingly automated and real-time monitoring of data is required during testing, and these data are presented in graphically intuitive manner. Based on this, this paper presents an implementation program, which uses cooperative working mode of upper computer and lower computer. Upper computer is mainly used to display real-time data, while lower computer mainly collects real-time data, and upper and lower computers uses modbus protocol for communication. UI part of upper computer is implemented using open-source cross-platform library Qt and extension library Qwt, with Qt Creator as development environment; lower computer mainly collects real-time data with microcontroller as basis. Upper and lower computers conduct cooperative work, able to display data in real time during testing.

\section{Overall Design}

\section{Background Architecture}

Detection of limestone $\mathrm{pH}$ and activity is a complex process. This process requires an environment with constant temperature. Traditional testing method is artificial titration. That is, in detection experiment, the sample to be tested in the cylinder needs artificial stir, while the experimental antacids are instilled, and lastly, $\mathrm{PH}$ test paper is used to measure $\mathrm{PH}$ value. The process requires manual operations, and antacids are slow in speed and low in flow in artificial titration experiments, and the amount of experimental acids cannot be precisely metered, resulting in greater experimental error, leading to inaccurate $\mathrm{PH}$ value and active value finally. In order to avoid errors caused by manual operations and to improve the detection accuracy, manual intervention needs to be minimized in the testing process, and full automation can be achieved in the entire testing process at best. Furthermore, temperature is also an important indicator in testing process. How to provide an experimental environment with constant temperature is an issue that needs to be considered by the system.

\section{Functional Requirements and Design}

In order to improve detection accuracy and to minimize manual steps, the detection system must meet the following functional requirements. Firstly, prior to detection, it needs to maintain an environment with constant temperature, and it is achieved by setting a thermostatic water tank, and the water tank has a capacity of about 2 liters, and there is a 2,000 $\mathrm{kW}$ heater tank inside. When the water temperature is below $43{ }^{\circ} \mathrm{C}$, the heater automatically starts, and when heated to $43^{\circ} \mathrm{C}$, the 
heating is stopped. When the water level in the tank is too low, it will trigger low water-level alarm device. In addition, the tank should also have insulation function. That is, when the water temperature is below 43 degrees, the heater will automatically turn on. When the water temperature reaches $43{ }^{\circ} \mathrm{C}$, and when the temperature is stable, experimental environment gets mature, and then the experiment can begin. At the beginning of the experiment, it firstly needs to inject $2000 \mathrm{ml}$ hot water to measuring glass, and the error of water amount cannot exceed $10 \mathrm{ml}$. In order to control precision of water injection, the total amount of water injection is calculated using Hall pulse meter to be about 5,000 pulses. After water injection is completed, the experiment can be started. During the experiment, the artificial stirring is changed to automatic mixing, and the agitator is driven by a motor. Start-stop of the motor can be controlled by a relay, while the relay can be controlled by software. During the experiment, the temperature should be maintained a constant temperature of $43{ }^{\circ} \mathrm{C}$. In order to maintain the temperature at $43{ }^{\circ} \mathrm{C}$, intermittent startup of heating means may be necessary. $\mathrm{PH}$ value of test samples in measuring glass is between 8 and 10. To maintain PH value between 8 and 10, rapid and timely injection of experiments antacids is required to precisely meter amount of instilled acid. Therefore, high-precision programmable peristaltic pump is employed to control injection of experimental acids, and via modbus protocol, the lower computer can send commands to the acid pump, taking control of the start-stop, rotational speed and rotational direction of acid and the like. After ten minutes, the experiment automatically comes to an end, and there is no need of human intervention during the experiment. After the end of the experiment, test reports can automatically generate.

Therefore, in order to meet the above requirements, the test system mainly achieves the following functional modules:

(1) Experimental tank temperature control module: it mainly controls the heating device of water tank, and controls operations of heater by Off -On of relay.

(2) Injection control module: it mainly controls water injection rate by controlling the Hall flowmeter with high requirements for precision, and positive-negative error of $2000 \mathrm{ml}$ water is $10 \mathrm{ml}$.

(3) Peristaltic pump control module: it mainly controls start-stop and revolving speed of peristaltic pump, thereby controlling the added amount of acid during the experiment. Total volume and flow of acids is required to be displayed in the UI in real time.

(4) Stirrer control module: it mainly controls start-stop of the agitator in experiment.

(5) Real-time graphics-rendering module: it mainly draws graphs of $\mathrm{pH}$ and activity in experiment.

(6) Experimental operating condition indication module: it mainly shows the experimental procedures.

(7) Experimental fault indication module: when the experiment fails, the module may indicate fault points.

System functional block diagram is shown in Figure 1

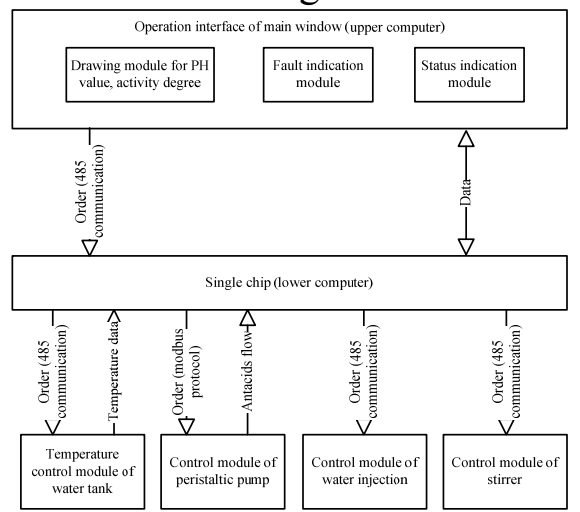

Fig.1. System functional block diagram

\section{Development Tools}

Qt is an open-source cross-platform development framework, and the program developed based 
on the framework can be seamlessly ported to any current mainstream PC platform and embedded platform, as long as Qt library of the same type and version is provided in the platform, and therefore, Qt is used to develop UI part. Qt Creator is mainly employed in development environment, and it is a Qt IDE closely combined with the development of Qt, able to set compiler and debugger, featured by highlighting syntax, automatic indentation, and auto-completion and so on. Qt library version chosen in the project is qt-everywhere-opensource-src-4.8.5.tar.gz, and Qt creator version is qt-creator-2.8.0-src.tar.gz. The operating system is fedora14.

Qwt is a Qt-based extension library, full name of Qt Widgets for Technical Applications, complying with the LGPL, and the source code is open. Qwt is mainly used for graphics rendering, able to draw graph curves in real time, various common mathematical function curves, as well as statistical graphs. These data are usually displayed in the form of 2D.

Modbus protocol is mainly used for mutual communication between the programmable controllers and can also be used to communication between devices on different networks. There are two communication modes, namely ASCII mode and RTU mode. When the controller conducts communication on the bus of Modbus in mode of ASCII, every eight bytes in a message is transmitted as two ASCII characters, and the main advantage of this model is that allowable time intervals between characters can be as long as IS, and the error does not occur too. Controller conducts communication on Modbus in RTU mode, and every eight bytes in a message are divided into two four hexadecimal characters, and the main advantage of this model is that at the same Baud rate, density of its transmitted characters is higher than ASCII mode, and each message must be transmitted continuously.

\section{Concrete Implementation}

Drawing module of real-time activity and $\mathrm{pH}$ is implemented using qwt library, and it is a key module of the detection system, requiring highlighting introduction; status indicator module, fault indication module and command and control module of the detection system are realized based on Qt library itself, and implementation method of several modules can be attributed to a class for introduction; communications between upper computer and lower computer as well as between lower computer and specific devices are primarily achieved through 485 serial port, involving modbus protocol and TCP protocol, which is introduced in third part.

(1) Realization of real-time drawing module

Real-time graphics drawing module consists of two parts, namely, real-time drawing module of activity and real-time drawing module of $\mathrm{pH}$. These two modules are implemented based on QwtPlot class in qwt library. QwtPlot class has integrated components such as canvas, axes and so on, and lattices, grids and curves can be drawn in line with needs. The module consists of the following three main areas:

Establishment of basic drawing components

When the basic drawing components that meet the requirements are established, following changes in the related properties of QwtPlot class need to be made,

SetAxisTitle (ui-u003e qwtPlotT-u003e yLeft, tr (" degree of activity ")) function sets the vertical axis label as " active degree",

SetAxisScale (ui-u003e qwtPlot-u003e y Left, 0,60,5) function sets the vertical scale between $0-60$, the interval is 5 , and horizontal axis label can be set to "time" with the same function, and the horizontal scale is $0-100$, interval of 10

By function set Canvas Background (Qt :: gray), the grid background is set to gray.

1) Drawing of real-time curve

Drawing of real-time curve requires using QwtPlotCurve class, and through receiving data of this class, real-time rendering graphics can be drawn on objects of QwtPlot class. Some properties of QwtPlot objects need to be set, and curve linearity is set through setstyle () function; setPen () function sets the color of curve; setRenderHint () can make the curve more smooth. After completion of property settings, data need to be added to the corresponding curves, and there are two coordinate values for each data, corresponding to the value of $\mathrm{X}$ and $\mathrm{Y}$ respectively. These two values are obtained from lower computer and saved in the QVector container of double type. 
setSamples () function in QwtPlotCurve class is called, and real-time drawing can be achieved after the obtained values of $\mathrm{X}$ and $\mathrm{Y}$ are added.

\section{2) Axis transformation}

The detection system has running time of 10 minutes, and a total of 1000 data points needs to be collected, namely, 100 data points per minute, while the maximum set on the horizontal axis is 1000 on the UI, then the drawing curved is too dense and difficult to observe, thus the maximum value drawn on the horizontal axis on the UI is 100 . When collection points reach integer multiples of 100 , drawing is re- started from the origin, but the coordinate value in the origin becomes the maximum value of the previous coordinates, and the like, until the drawing is completed. The program uses integer variable of tmpTxsize to conduct cumulative record of number of collected data points. When an integer multiple of 100 is reached, setAxisScale () function is called to re- set the $\mathrm{x}$ - axis scale.

(2) Implementation of operating control and parameter setting module

This module contains of several control acid pumps, mixers buttons, parameter setting panel and real-time operating status panel. Included qt library is mainly employed in implementation process, including QPushButton, QLCDNumber, WTextEdit, QGroupBox and other categories. When the control button is pressed, clicked () signal will trigger, and the system will automatically detect the slot function associated with the signal, and then executes the corresponding groove function.

(3) Implementation of communication module

The communication module contains two parts, namely, communication between upper computer and lower computer as well as between lower computer and specific equipment. Upper computer is PC, and lower computer is single-chip microcontroller. The communication between upper and lower computers need to use a hardware device, that is, 485 converter, and the converter can conduct mutual conversion between network signal and 485 data signal. Upper computer and the 485 converter are interconnected via cables. Then 485 converter can be seen as a small server, and upper computer is a client with respect to 485 converter, and they are communicated via TCP protocol; lower computer and 485 converter are interconnected via a serial line and are communicated via serial port. Commands sent by upper computer are forwarded to the 485 serial port after reaching 485 converter via TCP protocol and then sent to devices via serial cables. Control of stirrer and heater is relatively simple, while that of acid pump is more complex, for it requires turning to modbus protocol to generate commands in accordance with the agreement and to calibrate via CRC.

The running interface of entire software is shown in Figure 2:

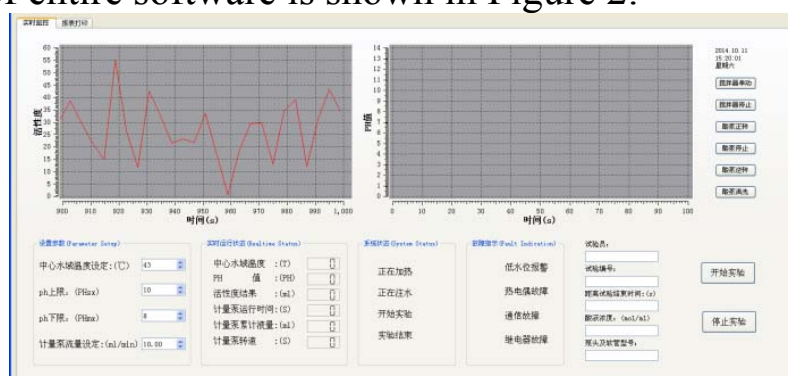

Fig.2. System running interface

\section{Conclusion}

Since Qt has good portability, Qt library and its extension library Qwt are employed for development, as long as the other platforms have Qt library of the same version, the software can be run directly. In addition, the communication between the lower machine and acid pump is achieved via modbus protocol, and communication commands are calibrated through the CRC to enhance accuracy of instruction. 


\section{References}

[1] Lu Huawei, Qin Pingjian and Zheng Rui. Design and implementation of Qt / Qwt-based operating monitoring system [J] Microcomputer Information. Volume 26 No.1-1, 2010: $72-74$

[2] Zhi-Ming Cai, Liu Chuanfu and Li Lixia , etc. Proficient Qt4 Programming [M] Beijing: Electronic Industry Press, 2008

[3] Blanchette J. Mark Summerfield C++ GUI Programming with Qt4[M].USA: Prentice Hall PTR, 2004.

[4 ] Feng Yaoling, Sun Yunjiang and Yao Aiqin. Design of QT-based electronic drawing board [J] Electronic Test, 2012, (12): 72-77.

[5] Huo Yafei. Explained development trainingof Qt and Qt Quick [M] Beijing: Beijing University of Aeronautics and Astronautics Press, 2012: 3-26.

[6] Zheng Archie and Chen Chao. Qt 4 Development training [M] Beijing: Electronic Industry Press, 2011: 100-199 .

[7] Lu Wenzhou .Qt5 development and examples [M] Beijing: Electronic Industry Press, 2013 : $1-116$.

[8] Zhang Bo. C ++ technology in Qt [M] Beijing: Electronic Industry Press, 2013 : 2-120. 